\title{
Review of: "Symmetry reduction in a hyperpolarization-activated homotetrameric ion channel"
}

\author{
Ingo Dreyer ${ }^{1}$ \\ 1 Universidad de Talca
}

Potential competing interests: The author(s) declared that no potential competing interests exist.

The article describes the cryo-EM structure of the plant K+ channel AKT1.

The introduction briefly but concisely describes the physiological background. Nevertheless, there are a few incorrectnesses to criticize:

*) line 36: "The Arabidopsis thaliana akt1 gene"; usually for plants, small letters are used to indicate nullallele plants. Genes are usually written as capital letters.

*) line 37: reference 6 is not correct. The correct reference for the identification of AKT1 is: doi: $10.1126 /$ science. 1585180

*) line 49: "patch clamp in X. laevis oocytes", the electrophysiological analyses were done with the twoelectrode voltage-clamp technique, not with the patch-clamp technique

*) line 51: references 8 and 10 would be appropriate instead of 8, only.

*) line 56: the appropriate reference for the described AKT1-AtKC1 heteromerization properties would be: doi: 10.1074/jbc.M109.017574

The structural analyses of AKT1 indicate that in particular the cytosolic C-terminal domains are rather flexible. They further point to interactions between a cytosolic N-terminal region and the C-linker right after the last transmembrane domain. Additionally, different symmetries of the tetrameric structure are observed. From the structural data interesting hypotheses were generated that might be tested in wet-lab experiments in future.

Point of criticism: Unfortunately, Figures S4 and S5 were mentioned in the text $(\mathrm{I} .46,101,104)$ but not available for review. 